\title{
ANALYTIC SOLUTIONS OF NONLINEAR COURNOT DUOPOLY GAME
}

\author{
AKIO MATSUMOTO AND MAMI SUZUKI
}

Received 12 December 2004

We construct a Cournot duopoly model with production externality in which reaction functions are unimodal. We consider the case of a Cournot model which has a stable equilibrium point. Then we show the existence of analytic solutions of the model. Moreover, we seek general solutions of the model in the form of nonlinear second-order difference equation.

\section{Introduction}

The main aim of this study is to construct an explicit form of an analytic solution of a multidimensional difference equation. To this end, we present a nonlinear Cournot duopoly model with unimodal reaction functions, show that its dynamical system is double logistic when the production externalities involved get stronger, and then give a constructive proof of the existence of an analytic solution of the dynamical system.

It is well known that there is an existence theorem for nonlinear differential equations, which guarantees the existence of an analytic solution. It is also well known that there is no general existence theorem for a nonlinear difference equation. In consequence, we check whether a discrete-time system to be considered has a solution or not, and then we examine its dynamical characteristic. In spite of this inconvenience, a discrete-time dynamical system is useful for analyzing economic phenomena observed in the real economic world such as business fluctuations that occur in real time. As far as global dynamics are concerned, the usual procedure is to perform numerical simulations to visualize how a dynamical process evolves over time, even if analytical treatment is insufficient. However, it is still possible that numerical simulations do not approximate the true behavior of a discrete-time system, mainly due to computer's storage limitations; truncations errors and the binary representation of numbers. To examine exact phenomena generated by a discrete-time system, it is therefore natural to attempt to provide its analytical solutions.

This study is organized as follows. Section 2 constructs a nonlinear duopoly model with unimodal reaction functions. The first half of Section 3 considers the existence of particular solutions. The second half, the main part of this study, seeks general analytic solutions and demonstrates their explicit forms. 


\section{Nonlinear duopoly model}

In this section, we present a discrete-time Cournot duopoly model with unimodal reaction functions. In a duopoly, where there are two firms, each firm has to take into account its rival's behavior, when it decides how much output to produce. In particular, we focus on the case in which each firm has to forecast the other firm's output choice. Given its forecast, each firm chooses a profit-maximizing output for itself. A Cournot equilibrium is a situation where each firm finds its beliefs about the other firm to be confirmed. We aim to construct an explicit form of an analytic solution approaching the Cournot equilibrium.

We begin by assuming that an inverse demand function is linear and decreasing,

$$
p=a-b Q, \quad a>0, b>0,
$$

where $Q$ is the industry output, provided that demand equals supply. Two firms, denoted by $X$ and $Y$, produce homogenous output $x$ and $y$ so that the total supply is made up of supplies from both firms, $x+y=Q$.

Let $x_{t}$ and $y_{t}$ be the outputs at time $t$. Firm $X$ expects that firm $Y$ will produce $y_{t}^{e}$ units of output where the $e$ stands for expected output. Then if firm $X$ decides to produce $x_{t}$ units of output, the total output it expects to be sold is $x_{t}+y_{t}^{e}$, which will yield a market price of $p_{t}=a-b\left(x_{t}+y_{t}^{e}\right)$. As for the production cost, we follow the formulation of Kopel [2] and focus on the case in which the production cost is assumed to incorporate positive production externality due the other firm's level of production. Denoting the production cost by $c^{x}\left(x_{t}, y_{t}^{e}, \alpha\right)$, firm $X$ solves the following optimization problem:

$$
\max _{x_{t}}\left\{\left[a-b\left(x_{t}+y_{t}^{e}\right)\right] x_{t}-c^{x}\left(x_{t}, y_{t}^{e}, \alpha\right)\right\},
$$

where

$$
c^{x}(x, y, \alpha)=d+a x-b x y(1+2 \alpha)+2 b \alpha x y^{2} .
$$

Its solution is called the firm's best-reply or reaction function. Differentiating the profit with respect to $x_{t}$ and setting it equal to zero gives the first-order condition which we can solve to derive the reaction function of firm $X$ :

$$
x_{t}=\alpha y_{t}^{e}\left(1-y_{t}^{e}\right) .
$$

Similarly, we can derive the reaction function of firm $Y$ :

$$
y_{t}=\beta x_{t}^{e}\left(1-x_{t}^{e}\right),
$$

where $c^{y}(y, x, \beta)=c^{x}(x, y, \alpha)$ is assumed. It gives the optimal choice of firm Y's output at time $t$ for a given expectation about firm X's output, $x_{t}^{e} . \alpha$ and $\beta$ are positive constants and measure the intensity of the positive externality.

A combination of output $\left(x^{c}, y^{c}\right)$ satisfying

$$
x^{c}=\alpha y^{c}\left(1-y^{c}\right), \quad y_{t}=\beta x^{c}\left(1-x^{c}\right)
$$


is a Cournot equilibrium in which each firm is maximizing its profits, given its expectations about the other firm's output choice, and, furthermore, these expectations are confirmed. In other words, each firm optimally chooses to produce the amount of output that the other firm expects it to produce.

To describe a process of adjustment to equilibrium, we introduce a formation of naive expectation, that is, $x_{t}^{e}=x_{t-1}$ and $y_{t}^{e}=y_{t-1}$. Namely, at the beginning of period $t$, one firm expects that the other will continue to keep its output at the same level as in the last period $t-1$. After substituting the values of expectations into the reaction functions, the time evolution of the duopoly system is represented by the double logistic discrete dynamical system:

$$
\mathrm{DS}_{1}:\left\{\begin{array}{l}
x_{t+1}=\alpha y_{t}\left(1-y_{t}\right), \\
y_{t+1}=\beta x_{t}\left(1-x_{t}\right)
\end{array}\right.
$$

A number of the Cournot equilibrium points depend on specified values of parameters, $\alpha$ and $\beta$. In particular, the dynamical system $D_{1}$ possesses only the trivial equilibrium $(0,0)$ when these parameters' values are less than unity. Beside the trivial equilibrium point, it has one positive equilibrium point when $\alpha$ and $\beta$ are greater than unity and less than or equal to three. It has three distinct equilibria when these are greater than three and less than or equal to four. It can generate a trajectory that can have negative values when either or both of $\alpha$ and $\beta$ are greater than four. The trivial equilibrium point is uninteresting. We have to solve the problem of selecting an equilibrium point values when the system yields multiple equilibrium point. Further, negative production are economically meaningless. Thus, in order to simplify our following analysis, we confine our attention to the case where there is only one positive Cournot equilibrium point. To do so, we make the following assumption.

Assumption 2.1. $1<\alpha<3$ and $1<\beta<3$.

To examine whether the Cournot equilibrium is locally stable, we linearize $\mathrm{DS}_{1}$ at the equilibrium point to form the Jacobi matrix,

$$
J=\left(\begin{array}{cc}
0 & \alpha\left(1-2 y^{c}\right) \\
\beta\left(1-2 x^{c}\right) & 0
\end{array}\right),
$$

where $\left(x^{c}, y^{c}\right)$ is the equilibrium point. (Substituting the second equation of $\mathrm{DS}_{1}$ into the first yields a quadratic equation in $x$ including the trivial solution. It is, thus, possible to have an explicit form of the equilibrium output, the form of which is, however, messy.) The eigenvalues $\lambda_{1}$ and $\lambda_{2}$ satisfy

$$
\begin{gathered}
\lambda_{1}+\lambda_{2}=0, \\
\lambda_{1} \lambda_{2}=-\alpha \beta\left(1-2 x^{c}\right)\left(1-2 y^{c}\right) .
\end{gathered}
$$

We put $\lambda=\lambda_{1}=-\lambda_{2}>0$. The Cournot equilibrium point is stable if $\lambda^{2}=\alpha \beta\left(1-2 x^{c}\right)(1-$ $\left.2 y^{c}\right)$ is less than unity. 


\section{Analytical solution}

Now we will look for an analytical solution of $\mathrm{DS}_{1}$ in this section. This section is divided into three subsections. We transform the dynamical system $\mathrm{DS}_{1}$ to a more manageable form $\mathrm{DS}_{3}$ in Section 3.1. We propose the existence of analytic particular solutions of the dynamical system in Section 3.2. We seek analytic general solutions in Section 3.3.

3.1. Transformation of variables. As a starting point for a more complicated study, we confine our analysis to the case in which the Cournot point is stable. The stable equilibrium point provides a convenient heuristic setting to detect analytical properties of the Cournot output adjustment; it makes the formidable mathematical problem simpler and more manageable; further, it enables us to derive rigorous results. Before proceeding to the system transformation, we make the following locally stable condition.

Assumption 3.1. $-1<-\alpha \beta\left(1-2 x^{c}\right)\left(1-2 y^{c}\right)<0$.

Assumption 3.1 leads to $|\lambda|<1$. Further, for technical reasons, we temporarily assume time $t$ to be a complex variable, although it is usually used as a real number. To emphasize this change, we hereafter adopt $x(t)$ to be the notation of variable dependency on time, rather than $x_{t}$. After we show the existence of analytic solutions, we treat $t$ as a real value to obtain a real value solution.

We transform the dynamical system $\mathrm{DS}_{1}$ to the equilibrium point by changing variables by $u(t)=x(t)-x^{c}$ and $v(t)=y(t)-y^{c}$ :

$$
\mathrm{DS}_{2}:\left\{\begin{array}{l}
u(t+1)=\alpha\left\{v(t)(1-v(t))+2 v(t) y^{c}\right\}, \\
v(t+1)=\beta\left\{u(t)(1-u(t))+2 u(t) x^{c}\right\} .
\end{array}\right.
$$

For the sake of simplicity, we further transform this simultaneous system to a secondorder difference equation. Let the first equation of $\mathrm{DS}_{2}$ shift one period ahead. Substituting the second equation into the shifted first equation yields a new dynamical system:

$$
\mathrm{DS}_{3}:\left\{\begin{array}{c}
u(t+2)=\alpha\left(1-2 y^{c}\right)\left\{\beta\left(1-2 x^{c}\right) u(t)-\beta u(t)^{2}\right\} \\
-\alpha \beta^{2}\left\{\left(1-2 x^{c}\right) u(t)-u(t)^{2}\right\}^{2} \\
v(t)=\Phi(u(t+1))=\frac{1-2 y^{c}}{2}\left\{1-\sqrt{1-\frac{4 \alpha u(t+1)}{\alpha\left(1-2 y^{c}\right)^{2}}}\right\} .
\end{array}\right.
$$

To show an existence of an analytic solution $u(t)$ of $\mathrm{DS}_{3}$, we further change variables by setting $s(t)=u(t)$ and $w(t)=u(t+1)$. 
3.2. Existence of an analytic solution. At first we can determine a formal solution of the first equation of $\mathrm{DS}_{3}$ such that

$$
u(t)=\sum_{n=1}^{\infty} a_{n} \lambda^{n t} .
$$

To determine coefficient values, $a_{k}$ of (3.3), we substitute (3.3) into the left-hand side of the first equation of $\mathrm{DS}_{3}$ and (3.3) with a two-period shift into the right-hand side. We then compare the coefficients of $\lambda^{n t}$ on both sides to find

$$
\begin{gathered}
a_{1} D(\lambda)=0, \\
a_{k} D\left(\lambda^{2 k}\right)=C_{k}\left(a_{1}, a_{2}, \ldots, a_{k-1}\right) \quad \text { for } k \geq 2,
\end{gathered}
$$

where $D(\lambda)=\lambda^{2}-\alpha \beta\left(1-2 y^{c}\right)\left(1-2 x^{c}\right)$ and $C_{k}$ polynomials of $a_{1}, a_{2}, \ldots, a_{k-1}$ are determined successively by calculation. (An illustrative example for determination of coefficients and constructions of $C_{k}$ is given in Appendix A.) By the definition of the characteristic equation, $D(\lambda)$ is zero, and thus we take $a_{1}$ to be arbitrarily but not zero. For $k \geq 2$, since $0<\lambda<1$ and $D\left(\lambda^{2 k}\right) \neq 0$, we can determine the coefficient $a_{k}$ with $a_{1}, a_{2}, \ldots, a_{k-1}$.

Next we change variables in $\mathrm{DS}_{3}$ by putting $s=u(t), w=u(t+1)$, and $z=u(t+2)$ and, for notational simplicity, define a new function of $s$ and $z$ in such a way that

$$
H(s, z)=-z+\alpha \beta\left(1-2 y^{c}\right)\left\{\left(1-2 x^{c}\right) s-s^{2}\right\}-\alpha \beta^{2}\left\{\left(1-2 x^{c}\right)^{2} s^{2}-2\left(1-2 x^{c}\right) s^{3}+s^{4}\right\} .
$$

Accordingly, the first equation of $\mathrm{DS}_{3}$ can be written as $H(s, z)=0$. Since $H(s, z)$ is holomorphic in a neighborhood of $z=0$, it can be checked that $H(0,0)=0$ and $\partial H(0,0) / \partial s$ $=-\lambda_{1} \lambda_{2}>0$. Thus we can have, with a help of the implicit function theorem, the holomorphic function $\phi$ such that for some $\rho>0$,

$$
s=\phi(z) \quad \text { for }|z| \leq \rho
$$

in which $\phi(0)=0$. Furthermore, we have a constant $K$ such that $|s|=|\phi(z)| \leq K|z|$ for $|z| \leq \rho$. We prove the existence of an analytic solution $u(t)$ such that $u(t)=\phi(u(t+2))$.

Given a positive integer $N$, we can define a partial sum of the formal solution by $P_{N}(t)=\sum_{n=1}^{N} \alpha_{n} \lambda^{n t}$. If a convergent analytic solution $u(t)$ of $\mathrm{DS}_{3}$ exists, then we are able to derive a convergent infinite series, $p_{N}(t)=u(t)-P_{N}(t)=O\left(\left|\lambda^{t}\right|^{N+1}\right)$. Conversely, if there exists a function $p_{N}(t)$ such that $p_{N}(t)+P_{N}(t)=\phi\left(p_{N}(t+2)+P_{N}(t+2)\right)$ and $p_{N}(t)=O\left(\left|\lambda^{t}\right|^{N+1}\right)$ for $\left|\lambda^{t}\right| \leq \eta_{N}$ with some $\eta_{N}>0$, then we can define a convergent series as a sum of $p_{N}(t)$ and $P_{N}(t), u(t)=p_{N}(t)+P_{N}(t)$, which can be an actual solution of $\mathrm{DS}_{3}$.

The steps of the proof are follows: (1) we show the existence of $p_{N}$ for $\left|\lambda^{t}\right| \leq \eta_{N}$ with some $\eta_{N}>0$ in Lemma 3.2; (2) we prove the uniqueness of $p_{N}$ in Lemma 3.3; (3) we demonstrate that the infinite series $p_{N}(t)+P_{N}(t)$ is independent from a choice of $N$ in Lemma 3.4. Here we put $p(t)=p_{N}(t), \eta=\eta_{N}$, and $p(t)=u(t)-P_{N}(t)$. 
124 Analytic solutions of nonlinear Cournot duopoly game

Now we define a set, such that

$$
S(\eta)=\left\{t \in C:\left|\lambda^{t}\right| \leq \eta\right\}
$$

Taking $A>0$ and $0<\eta$ which is determined later, we also define a set, such that

$$
J(A, \eta)=\left\{p: p(t) \text { is holomorphic and }|p(t)| \leq A\left|\lambda^{t}\right|^{N+1} \text { for } t \in S(\eta)\right\} .
$$

For $p(t) \in J(A, \eta)$, we further define map $T$ by

$$
T[p](t)=\phi\left(p(t+2)+P_{N}(t+2)\right)-P_{N}(t) .
$$

Lemma 3.2. Given $N$ and $\rho>0$, there exists a fixed point $p(t) \in J(A, \eta)$ of map $T$ for $t \in$ $S(\eta)$.

For the proof, see Appendix B.

We turn to the uniqueness of the fixed point.

Lemma 3.3. The fixed point $p(t) \in J(A, \eta)$ of $T$ is unique.

For the proof, see Appendix C.

Lemma 3.4 shows that the solution $u(t)$ does not depend on $N$.

Lemma 3.4. The solution $u(t)=p(t)+P_{N}(t)$ is independent of $N$.

For the proof, see Appendix D.

Lemmas 3.2, 3.3, and 3.4 prove that an analytic solution $u(t)$ of $\mathrm{DS}_{3}$ exists in $S(\eta)$ for an $\eta>0$, and has the expansion $u(t)=\sum_{n=1}^{\infty} a_{n} \lambda^{n t}$ as shown in (3.3). The function $\phi(z)$ in $s=\phi(z)$ for $|z| \leq \rho$ is defined only locally. Thus we have Theorem 3.5.

Theorem 3.5. Suppose Assumption 3.1, then there is a $\eta>0$ such that $\mathrm{DS}_{3}$ has an analytic solution $u(t)=\sum_{n=1}^{\infty} a_{n} \lambda^{n t}$ in $S(\eta)=\left\{t ;\left|\lambda^{t}\right|<\eta\right\}$.

However, the solution $u(t)$ can be analytically continued by keeping out of branch point. The solution $u(t)$ obtained is multivalued, because the function $\phi(z)$ in (3.6) is defined only locally.

Since $(-\lambda)^{k} \neq \lambda$ for any positive integer $k$ greater than 1 , then we can determine a formal solution $u_{2}(t)=\sum_{n=1}^{\infty} a_{2, n} \lambda^{n t}$ as in Section 3.2, with $-\lambda$ instead of $\lambda$. And it is shown to be an actual solution, as in the argument above. Therefore, putting $u_{1}(t)=u(t)$, $a_{1, n}=a_{n}$, and $\eta_{1}=\eta$, we have two analytic solutions $u_{1}(t)$ in $S\left(\eta_{1}\right)$ and $u_{2}(t)$ in $S\left(\eta_{2}\right)$.

3.3. General analytic solutions. This subsection is our main work. In this subsection, we seek general solutions of $\mathrm{DS}_{3}$.

For any linear second-order difference equation, general solutions are constructed from two particular solutions of it. But, for a nonlinear second-order difference equation, we can see that general solutions which converge to an equilibrium point of the equation are written from one of two particular solutions $u_{1}$ or $u_{2}$ of the difference equation. 
Here we put

$$
\begin{aligned}
l(x, y)= & \alpha\left(1-2 y^{c}\right)\left\{\beta\left(1-2 x^{c}\right) x-\beta x^{2}\right\} \\
& -\alpha \beta^{2}\left\{\left(1-2 x^{c}\right) x-x^{2}\right\}^{2}-\alpha \beta\left(1-2 x^{c}\right)\left(1-2 y^{c}\right) x .
\end{aligned}
$$

From $\mathrm{DS}_{3}$, we have

$$
u(t+2)=\alpha \beta^{2}\left\{\left(1-2 x^{c}\right) x-x^{2}\right\}^{2} u(t)+l(u(t), u(t+2)),
$$

where $l$ is higher-order term for $u(t), u(t+1)$.

Let $u(t)$ be a solution of $\mathrm{DS}_{3}$, and $w(t)=u(t+1)$. Then $\mathrm{DS}_{3}$ can be written as a system of simultaneous equations:

$$
\left(\begin{array}{l}
u(t+1) \\
w(t+1)
\end{array}\right)=\left(\begin{array}{cc}
0 & 1 \\
\alpha \beta\left(1-2 x^{c}\right)\left(1-2 y^{c}\right) & 0
\end{array}\right)\left(\begin{array}{l}
u(t) \\
w(t)
\end{array}\right)+\left(\begin{array}{c}
0 \\
l(u(t), w(t))
\end{array}\right) .
$$

Let $P=\left(\begin{array}{cc}1 & 1 \\ \lambda & -\lambda\end{array}\right)$ and put

$$
\left(\begin{array}{l}
u \\
w
\end{array}\right)=P\left(\begin{array}{c}
U \\
W
\end{array}\right)
$$

From $\lambda \neq-\lambda$, we can transform the coefficients matrix of linear terms of (3.12) into diagonal form, that is, (3.12) is transformed to the following system with respect to $U, W$ :

$$
\begin{gathered}
U(t+1)=\lambda U(t)+\sum_{i+j \geqq 2} c_{i j} U(t)^{i} W(t)^{j}=X(U(t), W(t)), \\
W(t+1)=-\lambda W(t)+\sum_{i+j \geqq 2} d_{i j} U(t)^{i} W(t)^{j}=Y(U(t), W(t)) .
\end{gathered}
$$

On the other hand, let $Q=\left(\begin{array}{cc}1 & 1 \\ -\lambda & \lambda\end{array}\right)$. Put

$$
\left(\begin{array}{l}
u \\
w
\end{array}\right)=Q\left(\begin{array}{c}
U \\
W
\end{array}\right)
$$

Then (3.12) is transformed to the following system with respect to $U, W$ :

$$
\begin{aligned}
& U(t+1)=-\lambda U(t)+\sum_{i+j \geq 2} c_{i j}^{\prime} U(t)^{i} W(t)^{j}=X^{\prime}(U(t), W(t)), \\
& W(t+1)=\lambda W(t)+\sum_{i+j \geq 2} d_{i j}^{\prime} U(t)^{i} W(t)^{j}=Y^{\prime}(U(t), W(t)) .
\end{aligned}
$$

From these transformation, we can obtain following relation.

Let $u(t)$ be a solution of $\mathrm{DS}_{3}$ in Section 3.2, and suppose $\Upsilon(t)$ is a solution of $\mathrm{DS}_{3}$ such that $\Upsilon(t+n) \rightarrow 0$ as $n \rightarrow+\infty$ uniformly on any compact subset of $t$-plane.

If a solution $(U(t), W(t))$ of (3.14) exists, then we obtain the inverse function $t=\psi(U)$ of $U=U(t)$ and we can write

$$
W(t)=W(\psi(U))=\Psi(U)
$$


126 Analytic solutions of nonlinear Cournot duopoly game

as far as $d U / d t \neq 0$. Then the function $\Psi$ satisfies

$$
\Psi(X(U, \Psi(U)))=Y(U, \Psi(U))
$$

where $X$ and $Y$ are defined in (3.14).

Conversely, we assume that a function $\Psi$ is a solution of the functional equation (3.18). If the first-order difference equation

$$
U(t+1)=X(U(t), \Psi(U(t)))
$$

has a solution $U(t)$, then we put $W(t)=\Psi(U(t))$ and have a solution $(U(t), W(t))$ of (3.14).

Put $\omega(t)=\Upsilon(t+1)$, and

$$
\left(\begin{array}{l}
\chi \\
\nu
\end{array}\right)=P^{-1}\left(\begin{array}{l}
\Upsilon \\
\omega
\end{array}\right)
$$

Then we have $\left(\begin{array}{l}\chi_{(t+n)}^{(t+n)} \\ \gamma(t+n)\end{array}\right) \rightarrow\left(\begin{array}{l}0 \\ 0\end{array}\right)$ since $\Upsilon(t+n) \rightarrow 0$ as $n \rightarrow 0$, and we obtain a solution $(\chi(t), \Psi(\chi(t)))$ of (3.14). According to $[4,5], \Psi$ is obtained, in a neighborhood of $x=0$, in the form

$$
\Psi(x)=\sum_{n=2}^{\infty} \gamma_{n} x^{n}
$$

that is, the expansion begins with $x^{2}$. From $\chi(t+1)=X(\chi(t), \Psi(\chi(t)))$, we have

$$
\frac{\chi(t+1)}{\chi(t)}=\lambda_{1}+\sum_{i+j \geqq 2} c_{i j} \chi(t)^{i-1} \Psi(\chi(t))^{j} .
$$

Since $\chi(t+n) \rightarrow 0$, as $n \rightarrow+\infty$ and by (3.21),

$$
\frac{\Psi(\chi(t+n))}{\chi(t+n)} \longrightarrow 0, \quad \frac{\chi(t+1+n)}{\chi(t+n)} \longrightarrow \lambda_{1}, \quad \text { as } n \longrightarrow+\infty .
$$

From $\Upsilon(t)=\chi(t)+\Psi(\chi(t))$, we have

$$
\begin{aligned}
\frac{\Upsilon(t+n+1)}{\Upsilon(t+n)} & =\frac{\chi(t+n+1) / \chi(t+n)+(\Psi(\chi(t+n+1)) / \chi(t+n+1)) \cdot(\chi(t+n+1) / \chi(t+n))}{1+\Psi(\chi(t+n)) / \chi(t+n)} \\
& \longrightarrow \lambda_{1}, \quad \text { as } n \longrightarrow+\infty .
\end{aligned}
$$

On the other hand, if a solution $(U, W)$ of (3.16) exists, and letting $\Psi$ be a solution of functional equation

$$
\Psi\left(X^{\prime}(U, \Psi(U))\right)=Y^{\prime}(U, \Psi(U))
$$


where $X^{\prime}$ and $Y^{\prime}$ are defined in (3.16), then we obtain similar relation of a solution $(U, W)$ of (3.16) and a solution $\Psi$ of (3.25). Furthermore, we have

$$
\frac{\Upsilon(t+1+n)}{\Upsilon(t+n)} \longrightarrow \lambda_{2}, \quad n \longrightarrow+\infty
$$

From the arguments above, we have following lemma.

Lemma 3.6. Suppose that $\Upsilon(t)$ is an analytic solution of $\mathrm{DS}_{3}$ such that $\Upsilon(t+n) \rightarrow 0$ as $n \rightarrow+\infty$, uniformly on any compact subset of $t$-plane, then there is the following dichotomy:

(I)

$$
\frac{\Upsilon(t+1+n)}{\Upsilon(t+n)} \longrightarrow \lambda_{1}, \quad n \longrightarrow+\infty
$$

(II)

$$
\frac{\Upsilon(t+1+n)}{\Upsilon(t+n)} \longrightarrow \lambda_{2}, \quad n \longrightarrow+\infty
$$

From Lemma 3.6, we have following theorem and we obtain general analytic solutions of $\mathrm{DS}_{3}$.

THEOREM 3.7. Let $\lambda_{1}$ and $\lambda_{2}$ be roots of the characteristic equation of $\mathrm{DS}_{1}$ and $0<\lambda=$ $\lambda_{1}=-\lambda_{2}<1$. Suppose that $u_{1}(\tau), u_{2}(\tau)$ are solutions of $\operatorname{DS}_{3}$ which have expansions $u_{1}(t)=$ $\sum_{n=1}^{\infty} a_{1, n} \lambda_{1}^{n t}$ in $S\left(\eta_{1}\right)=\left\{t ;\left|\lambda_{1}^{t}\right|<\eta_{1}\right\}, u_{2}(t)=\sum_{n=1}^{\infty} a_{2, n} \lambda_{2}^{n t}$ in $S\left(\eta_{2}\right)=\left\{t ;\left|\lambda_{2}^{t}\right|<\eta_{2}\right\}$, with some constant $\eta_{1}, \eta_{2}>0$. Further, suppose that $\Upsilon(t)$ is an analytic solution of $\mathrm{DS}_{3}$ such that $\Upsilon(t+n) \rightarrow 0$ as $n \rightarrow+\infty$, uniformly on any compact subset of $t$-plane. If the solution $\Upsilon$ of $\mathrm{DS}_{3}$ satisfies $\Upsilon(t+1+n) / \Upsilon(t+n) \rightarrow \lambda_{i},(i=1,2)$, as $n \rightarrow+\infty$, then there is a periodic entire function $\pi_{i}(t),\left(\pi_{i}(t+1)=\pi_{i}(t)\right)$, such that

$$
\begin{aligned}
\Upsilon(t)= & \frac{1}{\lambda_{i+1}-\lambda_{i}}\left(\sum_{n=1}^{\infty} a_{i, n} \lambda_{i}^{n\left(t+\pi_{i}(t)\right)}-\sum_{n=1}^{\infty} a_{i, n} \lambda_{i}^{n\left(t+\pi_{i}(t)+1\right)}\right) \\
& +\Psi_{i}\left(\frac{1}{\lambda_{i+1}-\lambda_{i}}\left(\sum_{n=1}^{\infty} a_{i, n} \lambda_{i}^{n\left(t+\pi_{i}(t)\right)}-\sum_{n=1}^{\infty} a_{i, n} \lambda_{i}^{n\left(t+\pi_{i}(t)+1\right)}\right)\right),
\end{aligned}
$$

in $S\left(\eta_{i}\right)$, with the convention $\lambda_{3}$ means $\lambda_{1}$. When $i=1, \Psi_{1}$ is a solution of (3.18). When $i=2, \Psi_{2}$ is a solution of (3.25) in which $X, Y$ are defined in (3.14), and $X^{\prime}, Y^{\prime}$ are defined in (3.16).

Conversely, a function $\Upsilon(t)$ which is represented as shown in (3.29) in $S\left(\eta_{i}\right)$ for some $\eta_{i}>$ 0 , where $\pi_{i}(t)$ is a periodic function with period 1 , is a solution of $\mathrm{DS}_{3}$ such that $\Upsilon(t+n) \rightarrow 0$ and $\Upsilon(t+1+n) / \Upsilon(t+n) \rightarrow \lambda_{i}$ as $n \rightarrow+\infty$ with $i=1,2$.

Proof. Here we prove case (I), when $i=1$. Put $u(t)=u_{1}(t)$ to be the solution of $\mathrm{DS}_{3}$ which we obtain in Theorem 3.5 as $u(t)=\sum_{n=1}^{\infty} a_{n} \lambda^{n t},\left(a_{n}=a_{1, n}\right)$. And suppose $\Upsilon(t)$ is a solution of $\mathrm{DS}_{3}$ such that $\Upsilon(t+n) \rightarrow 0$ as $n \rightarrow+\infty$ uniformly on any compact set. 
Put $\omega(t)=\Upsilon(t+1)$, and $\left(\begin{array}{l}\chi \\ \nu\end{array}\right)=P^{-1}\left(\begin{array}{c}\Upsilon \\ \omega\end{array}\right)$, where $(\chi, \nu)$ is a solution of (3.14). Then we have

$$
U(t)=\frac{1}{\lambda_{2}-\lambda}\left(\lambda_{2} u(t)-u(t+1)\right)=\frac{1}{\lambda_{2}-\lambda}\left(\sum_{n=1}^{\infty}\left(\lambda_{2} a_{n}-a_{n} \lambda^{n}\right)\left(\lambda^{t}\right)^{n}\right)=\tilde{U}\left(\lambda^{t}\right),
$$

where $\zeta=\tilde{U}(\tau)$ is a function of $\tau=\lambda^{t}$ and $\tilde{U}^{\prime}(0)=a_{1} \neq 0$ and $\tilde{U}(0)=0$. Since $\tilde{U}(\tau)$ is an open map, for any $\eta_{1}>0$, there is an $\eta_{2}>0$ such that $\tilde{U}\left(\left\{|\tau|<\eta_{1}\right\}\right) \supset\left\{|\zeta|<\eta_{2}\right\}$. Since $\chi(t+n) \rightarrow 0$ as $n \rightarrow \infty$, supposed that $t$ belongs to a compact set $K$, there is an $n_{0} \in \mathbb{N}$ such that for $t^{\prime} \in K,\left|\chi\left(t^{\prime}+n\right)\right|<\eta_{2}\left(n \geqq n_{0}\right)$.

Thus there is a $\tau^{\prime}=\lambda^{\sigma}$, such that

$$
\chi\left(t^{\prime}+n\right)=\tilde{U}\left(\tau^{\prime}\right)=\tilde{U}\left(\lambda^{\sigma}\right) .
$$

Since $\tilde{U}^{\prime}(0)=a_{1} \neq 0$, using the theorem on implicit function for (3.31), we have $\tilde{U}^{-1}$ such that $\lambda^{\sigma}=\tilde{U}^{-1}\left(\chi\left(t^{\prime}+n\right)\right)$. Put $t=t^{\prime}+n$, then $\lambda^{\sigma}=\tilde{U}^{-1}(\chi(t))$, and we write $\sigma=$ $\log _{\lambda} \tilde{U}^{-1}(\chi(t))=\tilde{l}(t)$.

When there is a solution $\chi(t)$ of (3.14), we have

$$
\begin{aligned}
\chi(t+1) & =X(\chi(t), \Psi(\chi(t)))=X\left(\tilde{U}\left(\lambda^{\sigma}\right), \Psi\left(\tilde{U}\left(\lambda^{\sigma}\right)\right)\right) \\
& =X(U(\sigma), \Psi(U(\sigma)))=U(\sigma+1)=\tilde{U}\left(\lambda^{\sigma+1}\right) .
\end{aligned}
$$

Therefore, we have $\sigma+1=l(t+1), l(t)+1=l(t+1)$. If we put $\pi_{1}(t)=\ell(t)-t$, then we obtain $\pi_{1}(t+1)=\ell(t+1)-(t+1)=\ell(t)-t=\pi_{1}(t)$, and we can write $\pi_{1}(t)$ defined for a compact set $K$ with $\mathfrak{R}[t]$ sufficiently large, which we can continue analytically as a periodic function with period 1 , as

$$
l(t)=t+\pi_{1}(t)
$$

then $\sigma=t+\pi_{1}(t)$.

From (3.30) and (3.31), $\chi(t)$ can be written as $\chi(t)=\tilde{U}\left(\lambda^{t+\pi_{1}(t)}\right)=U\left(t+\pi_{1}(t)\right)=$ $\left(1 /\left(\lambda_{2}-\lambda_{1}\right)\right)\left(\lambda_{2} u\left(t+\pi_{1}(t)\right)-u\left(t+1+\pi_{1}(t)\right)\right)$. Using (3.31), we have

$$
\begin{aligned}
\Upsilon(t)= & \chi(t)+\nu(t) \\
= & \chi(t)+\Psi(\chi(t)) \\
= & U\left(t+\pi_{1}(t)\right)+\Psi\left(U\left(t+\pi_{1}(t)\right)\right) \\
= & \frac{1}{\lambda_{2}-\lambda_{1}}\left(\sum_{n=1}^{\infty} a_{n} \lambda^{n\left(t+\pi_{1}(t)\right)}-\sum_{n=1}^{\infty} a_{n} \lambda^{n\left(t+\pi_{1}(t)+1\right)}\right) \\
& +\Psi\left(\frac{1}{\lambda_{2}-\lambda_{1}}\left(\sum_{n=1}^{\infty} a_{n} \lambda^{n\left(t+\pi_{1}(t)\right)}-\sum_{n=1}^{\infty} a_{n} \lambda^{n\left(t+\pi_{1}(t)+1\right)}\right)\right),
\end{aligned}
$$

where $\Psi$ is a solution of (3.18) as shown in (3.21) and $\pi_{1}$ is defined for $t \in \cup_{n \in \mathbb{Z}}(K+n)$ with a compact set $K$. Since $K$ is arbitrary, we can continue $\pi(t)$ analytically as periodic 
entire function with period 1. From Lemma 3.6, we have $\Upsilon(t+1+n) / \Upsilon(t+n) \rightarrow \lambda_{1}, n \rightarrow$ $+\infty$.

Conversely, if we put $\Upsilon(t)$ such that (3.29), where $\pi$ is an arbitrary periodic entire function, and $\Psi$ is a solution of (3.18), then we can have $\Upsilon(t)$ as a solution of $\mathrm{DS}_{3}$ such that $\Upsilon(t+n) \rightarrow 0$ as $n \rightarrow+\infty$. Furthermore, we have a solution $\chi$ of (3.14) such that

$$
\Upsilon(t)=\chi(t)+\Psi(\chi(t))
$$

where $\chi(t+n) \rightarrow 0$ as $n \rightarrow+\infty$. Hence we have $\Upsilon(t+1+n) / \Upsilon(t+n) \rightarrow \lambda_{1}$ as $n \rightarrow+\infty$.

For case (II), when $i=2$, the proof is similar to the above proof with (3.15) and (3.25).

From Theorem 3.7, general analytic solutions of $\mathrm{DS}_{3}$ which converge to 0 are given.

Thus we can obtain analytic general solutions $(x(t), y(t))$ of $\mathrm{DS}_{1}$ which converge to $\left(x^{c}, y^{c}\right)$ as follow:

$$
x(t)=\Upsilon(t)+x^{c}, \quad y(t)=\Phi(\Upsilon(t))+y^{c} .
$$

\section{Appendices}

\section{A.}

In this appendix, we outline the procedure to determine values of coefficients $a_{n}$.

$\mathrm{DS}_{3}$ is spelled out as

$$
\begin{aligned}
u(t+2)= & \alpha \beta\left(1-2 x^{c}\right)\left(1-2 y^{c}\right) u(t)-\alpha \beta\left\{\left(1-2 y^{c}\right)+\beta\left(1-2 x^{c}\right)^{2}\right\} u(t)^{2} \\
& +2 \alpha \beta^{2}\left(1-2 x^{c}\right) u(t)^{3}-\alpha \beta^{2} u(t)^{4} .
\end{aligned}
$$

By the definition of the formal solution, $u(t)=\sum_{n=1}^{\infty} a_{n} \lambda^{n t}$ and $u(t+2)=\sum_{n=1}^{\infty} a_{n} \lambda^{n(t+2)}$, which are substituted into the above equation,

$$
\begin{aligned}
a_{1} \lambda^{2} \lambda^{t}+ & a_{2} \lambda^{4} \lambda^{2 t}+a_{3} \lambda^{6} \lambda^{3 t}+\cdots \\
= & \alpha \beta^{2}\left(1-2 x^{c}\right)\left(1-2 y^{c}\right)\left\{a_{1} \lambda^{t}+a_{2} \lambda^{2 t}+a_{3} \lambda^{3 t}+\cdots\right\} \\
& -\alpha \beta\left\{\left(1-2 y^{c}\right)+\beta\left(1-2 x^{c}\right)^{2}\right\}\left\{a_{1} \lambda^{t}+a_{2} \lambda^{2 t}+a_{3} \lambda^{3 t}+\cdots\right\}^{2} \\
& +2 \alpha \beta^{2}\left(1-2 x^{c}\right)\left\{a_{1} \lambda^{t}+a_{2} \lambda^{2 t}+a_{3} \lambda^{3 t}+\cdots\right\}^{3} \\
& -\alpha \beta^{2}\left\{a_{1} \lambda^{t}+a_{2} \lambda^{2 t}+a_{3} \lambda^{3 t}+\cdots\right\}^{4} .
\end{aligned}
$$

We compare the coefficients of $\lambda^{\text {nt }}$ in the left hand side of the above equation with the ones in the right hand side. For the coefficients of $\lambda^{t}$, we have

$$
a_{1}\left\{\lambda^{2}-\alpha \beta\left(1-2 x^{c}\right)\left(1-2 y^{c}\right)\right\}=a_{1} D(\lambda),
$$

where $D(\lambda)=\lambda^{2}-\alpha \beta\left(1-2 x^{c}\right)\left(1-2 y^{c}\right)$ is the characteristics polynomial of $\mathrm{DS}_{3}$. Since $D(\lambda)=0$ identically, $a_{1}$ can be arbitrary. For the coefficients of $\lambda^{2 t}$, we have

$$
a_{2} D\left(\lambda^{2}\right)=-\alpha\left\{\beta\left(1-2 y^{c}\right)+\alpha^{2}\left(1-2 x^{c}\right)^{2}\right\} a_{1}^{2} .
$$




\section{Analytic solutions of nonlinear Cournot duopoly game}

Since $\lambda^{2} \neq \lambda_{1}, \lambda_{2}$, we have $D\left(\lambda^{k}\right) \neq 0(k \geq 2)$. So we can determine $a_{2}$ by dividing both sides by $D\left(\lambda^{2}\right)$,

$$
a_{2}=\frac{C_{2}\left(\alpha_{1}\right)}{D\left(\lambda^{2}\right)},
$$

where $C_{2}\left(\alpha_{1}\right)=-\alpha\left\{\beta\left(1-2 y^{c}\right)+\alpha^{2}\left(1-2 x^{c}\right)^{2}\right\} a_{1}^{2}$. By the same token, for the coefficients of $\lambda^{3 t}$, we have

$$
a_{3} D\left(\lambda^{3}\right)=-2 \alpha\left\{\left(\beta\left(1-2 y^{c}\right)+\alpha^{2}\left(1-2 x^{c}\right)^{2}\right) a_{1} a_{2}-\alpha \beta\left(1-2 x^{c}\right) a_{1}^{3}\right\}
$$

and then

$$
a_{3}=\frac{C_{3}\left(a_{1}, a_{2}\right)}{D\left(\lambda^{3}\right)}
$$

where $C_{3}\left(a_{1}, a_{2}\right)=-2 \alpha\left\{\left(\beta\left(1-2 y^{c}\right)+\alpha^{2}\left(1-2 x^{c}\right)^{2}\right) a_{1} a_{2}-\alpha \beta\left(1-2 x^{c}\right) a_{1}^{3}\right\}$. Similarly, for the coefficients of $\lambda^{n t}(n \geq 4), \alpha_{n}$ is sequentially determined by $a_{1}, a_{2}, \ldots, a_{n-1}$ by

$$
a_{n}=\frac{C_{n}\left(a_{1}, a_{2}, \ldots a_{n-1}\right)}{D\left(\lambda^{n}\right)},
$$

where $C_{n}\left(a_{1}, a_{2}, \ldots, a_{n-1}\right)$ is defined accordingly.

\section{B. Proof of Lemma 3.2}

In this appendix, we prove Lemma 3.2. Since $\phi$ is holomorphic on $|z| \leq \rho$, we have

$$
\frac{d}{d z} \phi(z)=\frac{1}{2 \pi i} \int_{|\xi|=\rho} \frac{\phi(\xi)}{(\xi-z)^{2}} d \xi
$$

When $|z| \leq \rho / 2$, we have $|\xi-z| \geq|\xi|-|z| \geq \rho-\rho / 2=\rho / 2$, and hence

$$
\left|\frac{d}{d z} \phi(z)\right| \leq \frac{1}{\pi} \int_{|\xi|=\rho} \frac{|\phi(\xi)|}{(\rho / 2)^{2}} d \xi \leq \frac{1}{\pi} \int_{|\xi|=\rho} \frac{K}{(\rho / 2)^{2}} d \xi=\frac{8 K}{\rho} .
$$

Next we choose $A$ and $\eta$ such that $A \eta^{N+1}<\rho / 4$. Then for sufficiently large $t$, we have $|p(t)| \leq A\left|\lambda^{t}\right|^{N+1} \leq A \eta^{N+1}<\rho / 4$. The inequality still holds even for $t+2$,

$$
|p(t+2)| \leq A\left|\lambda^{t+2}\right|^{N+1}=A|\lambda|^{2(N+1)}\left|\lambda^{t}\right|^{N+1}<\frac{\rho}{4} .
$$

Consequently, for $t$ large enough, $\left|P_{N}(t+2)\right|<\rho / 4$, and then

$$
|z|=\left|p(t+2)+P_{N}(t+2)\right| \leq \frac{\rho}{4}+\frac{\rho}{4}=\frac{\rho}{2} .
$$

Since

$$
g_{1}(t, p(t+2))=\int_{0}^{1} p(t+2) \frac{d}{d r} \phi\left(r p(t+2)+P_{N}(t+2)\right) d r
$$


(B.2), (B.3), and (B.4) imply

$$
\left|g_{1}(t, p(t+2))\right| \leq \frac{8 K}{\rho} A|\lambda|^{N+1}\left|\lambda^{t}\right|^{N+1} .
$$

From the definition of $g_{2}$, we have

$$
\left|g_{2}(t)\right| \leq K_{2}\left|\lambda^{t}\right|^{N+1}
$$

where $K_{2}$ is a constant but its magnitude depends on $N$. Hence, using (B.6) and (B.7), we have

$$
|T[p](t)| \leq\left|g_{1}(t, p(t+1), p(t+2))\right|+\left|g_{2}(t)\right| \leq\left(\frac{8 K}{\rho} A|\lambda|^{N+1}+K_{2}\right)\left|\lambda^{t}\right|^{N+1} .
$$

If we suppose $N$ is so large that $(8 K / \rho)|\lambda|^{N+1}<1 / 2$, moreover we take $A$ to be so large that $A>2 K_{2}$, then we obtain

$$
|T[p](t)| \leq A\left|\lambda^{t}\right|^{N+1}
$$

Hence, we find that $T$ maps $J(A, \eta)$ into itself. The map $T$ is obviously continuous if $J(A, \eta)$ is endowed with topology of uniform convergence on a compact set in $S(\eta)$. $J(A, \eta)$ is clearly convex and is relatively compact due to the theorem of Montel [1]. Since requirements of Schauder's fixed point theorem [3] are all satisfied, we can show the existence of a fixed point $p(t) \in J(A, \eta)$ of $T$ which depends on $N$. This proves Lemma 3.2.

\section{Proof of Lemma 3.3}

In this appendix, we prove Lemma 3.3. Suppose that there exists another fixed point, $p^{*}(t) \in J\left(A^{*}, \eta^{*}\right)$. Put $A_{0}=\max \left(A, A^{*}\right), \eta_{0} \leq \min \left(\eta, \eta^{*}\right), u(t)=p(t)+P_{N}(t), u^{*}(t)=$ $p^{*}(t)+P_{N}(t)$, and $q(t)=p^{*}(t)-p(t)$, then we have $|q(t)| \leq 2 A_{0}$ and

$$
\begin{aligned}
q(t)= & \left\{\phi\left(p^{*}(t+2)+P_{N}(t+2)\right)-\phi\left(P_{N}(t+2)\right)-P_{N}(t)\right\} \\
& -\left\{\phi\left(p(t+2)+P_{N}(t+2)\right)-\phi\left(P_{N}(t+2)\right)-P_{N}(t)\right\} \\
= & \phi(q(t+2)+u(t+2))-\phi(u(t+2)) \\
= & \int_{0}^{1} q(t+2) \frac{d}{d z} \phi(r q(t+2)+u(t+2)) d r .
\end{aligned}
$$

If $\eta_{0}$ is sufficiently small, then

$$
\left|\frac{d}{d z} \phi(r q(t+2)+u(t+2))\right|<\frac{8 K}{\eta}, \quad|\lambda|^{N+1}<\frac{\eta}{32 K} .
$$

Consequently, we have

$$
|q(t)| \leq \int_{0}^{1} \frac{8 K}{\rho}|q(t+2)| d r \leq \int_{0}^{1} \frac{8 K}{\rho}|\lambda|^{N+1}\left(2 A_{0}\left|\lambda^{t}\right|^{N+1}\right) d r<\frac{1}{2} A_{0}\left|\lambda^{t}\right|^{N+1} .
$$


132 Analytic solutions of nonlinear Cournot duopoly game

Hence,

$$
|q(t)|=\left|p^{*}(t)-p(t)\right| \leq \frac{1}{2} A_{0}\left|\lambda^{t}\right|^{N+1}=\left(\frac{1}{4}\right) 2 A_{0}\left|\lambda^{t}\right|^{N+1}
$$

Next we consider $q(t)$ in which $|q(t)| \leqq(1 / 4) \cdot 2 A_{0}\left|\lambda^{t}\right|^{N+1}$ and repeat this procedure, then we have $|q(t)| \leqq(1 / 4)^{2} \cdot 2 A_{0}\left|\lambda^{t}\right|^{N+1}$. Repeating this procedure $k$ times, we obtain

$$
\left|p^{*}(t)-p(t)\right|<\left(\frac{1}{4}\right)^{k}\left(2 A_{0}\right)\left|\lambda^{t}\right|^{N+1}, \quad k=1,2, \ldots
$$

where the inequality holds for any $k$. Letting $k \rightarrow \infty$, we have

$$
p^{*}(t)=p(t) \quad \text { for } t \in S\left(\eta_{0}\right)
$$

Since this implies that $p^{*}(t)=p(t)$ and $p(t)=p(t)$ are holomorphic in $\left|\lambda^{t}\right| \leq \min \left(\eta, \eta^{*}\right)$, we conclude, $p^{*}(t) \equiv p(t)$. This completes the proof of Lemma 3.3.

\section{Proof of Lemma 3.4}

In this appendix, we prove Lemma 3.4. Let $p_{N}(t) \in J\left(A_{N}, \eta_{N}\right)$ and $p_{N+1}(t) \in$ $J\left(A_{N+1}, \eta_{N+1}\right)$ be fixed points of $T$, and

$$
u_{N+1}(t)=p_{N+1}(t)+P_{N+1}(t)=p_{N+1}(t)+a_{N+1} \lambda^{(N+1) t}+P_{N}(t)=\tilde{p}_{N}(t)+P_{N}(t) .
$$

Then we have

$$
\begin{aligned}
\left|\tilde{p}_{N}(t)\right| & =\left|p_{N+1}(t)+a_{N+1} \lambda^{(N+1) t}\right| \\
& \leq A_{N+1} \lambda^{N+2}\left|\lambda^{t}\right|^{N+2}+\left|a_{N+1}\right|\left|\lambda^{t}\right|^{N+1} \\
& =\left(A_{N+1}|\lambda|+\left|a_{N+1}\right|\right)\left|\lambda^{t}\right|^{N+1} \\
& =A_{N}^{*}\left|\lambda^{t}\right|^{N+1} .
\end{aligned}
$$

By the uniqueness of the fixed point, $\tilde{p}_{N}(t)=p_{N}(t)$ for $t \in S\left(\eta_{N}\right) \cap S\left(\eta_{N+1}\right)$. Thus

$$
u_{N+1}(t)=u_{N}(t) \quad \text { for } t \in S\left(\eta_{N}\right) \cap S\left(\eta_{N+1}\right)
$$

By analytic prolongation [1], both $u_{N}(t)$ and $u_{N+1}(t)$ are holomorphic in $S\left(\eta_{N}\right) \cup S\left(\eta_{N+1}\right)$ and coincide. This completes the proof of Lemma 3.4.

\section{Acknowledgment}

This research was partially supported by the Grant-in-Aid for Scientific Research (C) 15540217 from the Ministry of Education, Science, and Culture, Japan. 


\section{References}

[1] L. V. Ahlfors, Complex Analysis, McGraw-Hill, New York, 1996.

[2] M. Kopel, Simple and complex adjustment dynamics in Cournot duopoly models, Chaos Solitons Fractals 7 (1996), no. 12, 2031-2048.

[3] D. R. Smart, Fixed Point Theorems, Cambridge Tracts in Mathematics, no. 66, Cambridge University Press, New York, 1974.

[4] M. Suzuki, Holomorphic solutions of some functional equations, Nihonkai Math. J. 5 (1994), no. $2,109-114$.

[5]__ Holomorphic solutions of some system of $n$ functional equations with $n$ variables related to difference systems, Aequationes Math. 57 (1999), no. 1, 21-36.

Akio Matsumoto: Department of Economics, Chuo University, 742-1 Higashinakano, Hachiojishi, Tokyo 192-0393, Japan

E-mail address: akimo@tamacc.chuo-u.ac.jp

Mami Suzuki: Department of Management Informatics, Aichi Gakusen University, 1 Shiotori, Oike-cho, Toyota, 474-8532, Japan

E-mail address: m-suzuki@gakusen.ac.jp 


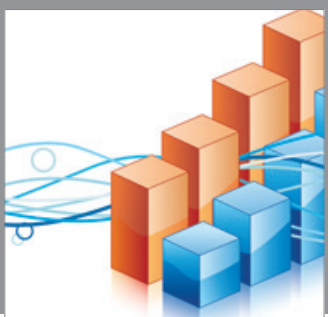

Advances in

Operations Research

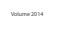

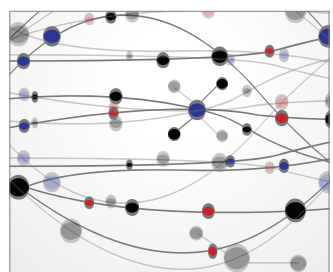

\section{The Scientific} World Journal
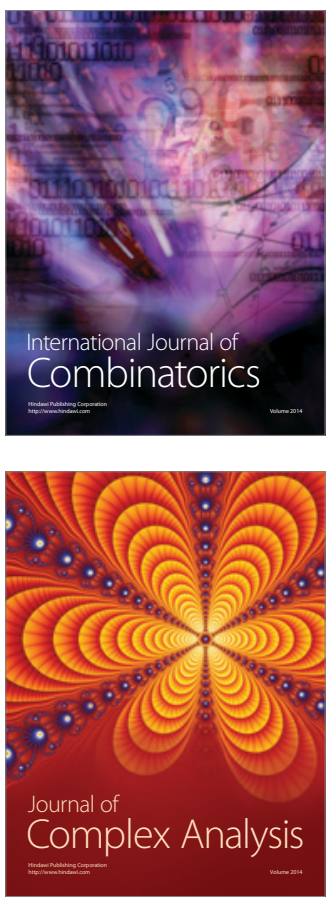

International Journal of

Mathematics and

Mathematical

Sciences
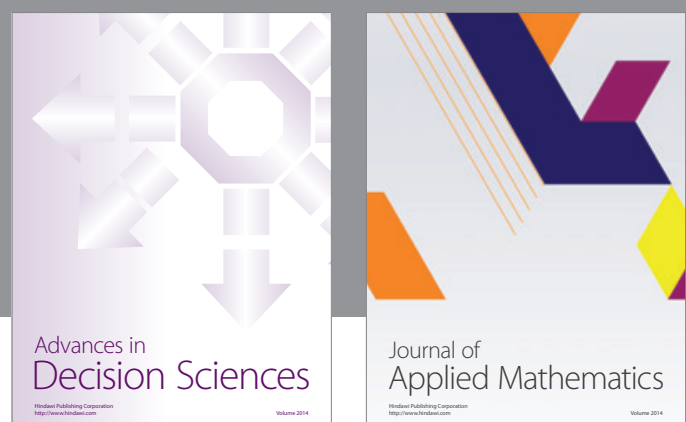

Journal of

Applied Mathematics
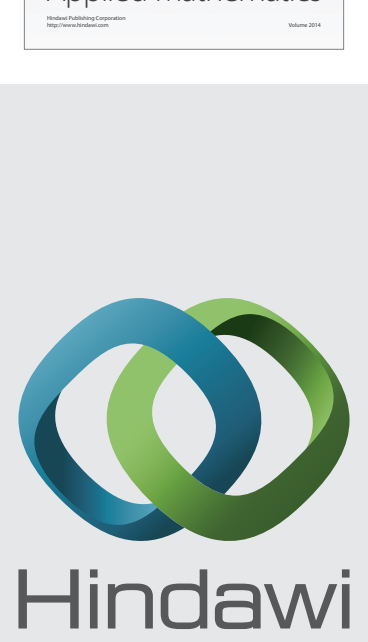

Submit your manuscripts at http://www.hindawi.com
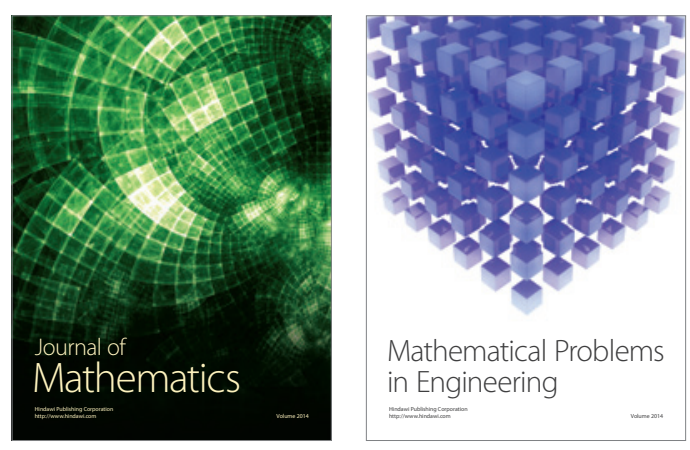

Mathematical Problems in Engineering
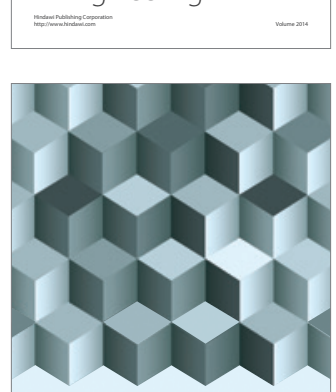

Journal of

Function Spaces
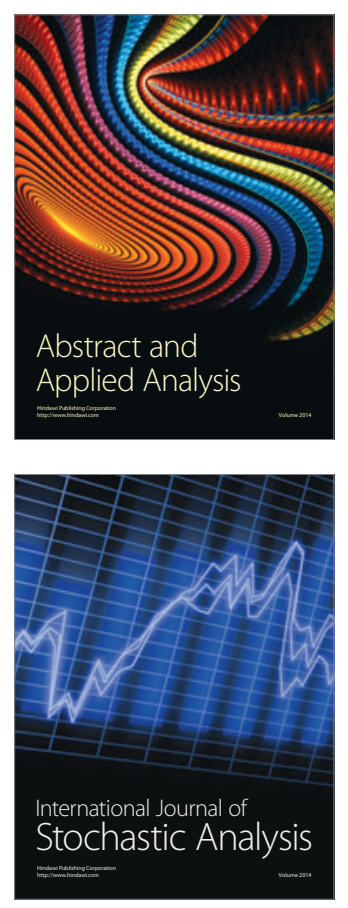

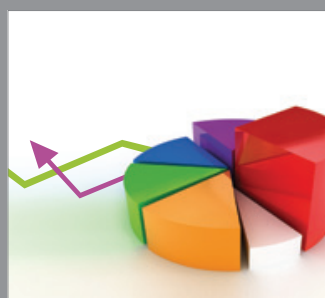

ournal of

Probability and Statistics

Promensencen
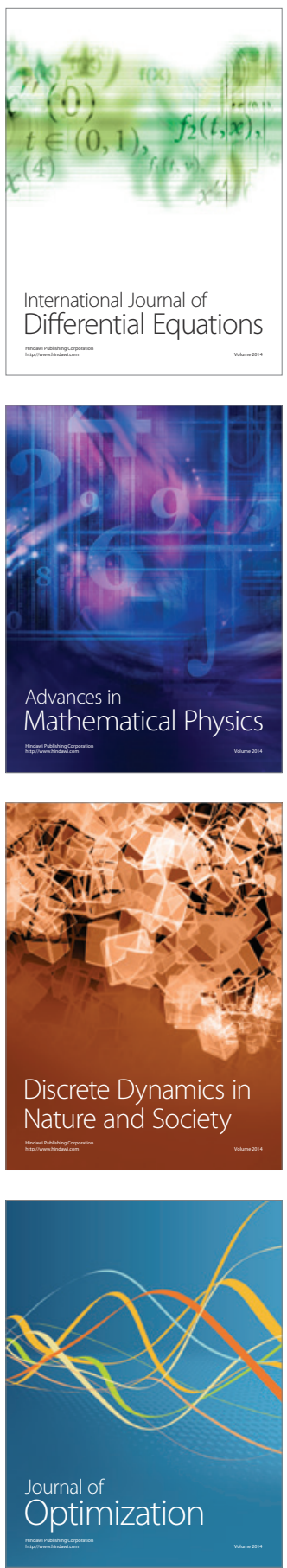